\title{
Classificação Inteligente de Eventos Extremos Utilizando Redes Neurais e HOG em Imagens de Satélite
}

\author{
Luís Ricardo Arantes Filho ${ }^{1}$ \\ Instituto Nacional de Pesquisas Espaciais \\ Émerson Jean da Silva ${ }^{2}$ \\ Instituto Nacional de Pesquisas Espaciais \\ Francisca Joamila Brito do Nascimento ${ }^{3}$ \\ Instituto Nacional de Pesquisas Espaciais \\ Lamartine Nogueira Frutuoso Guimarães ${ }^{4}$ \\ Instituto de Estudos Avançados \\ Reinaldo Roberto Rosa ${ }^{5}$ \\ Instituto Nacional de Pesquisas Espaciais
}

Resumo. Este trabalho apresenta uma proposta de análise de eventos extremos em imagens de satélite, especificamente a caracterização de eventos climáticos como chuvas torrenciais, tempestades, furacões e tornados. A classificação de eventos climáticos é de certa forma, algo de grande importância para todas as categorias sociais e governamentais, isto se dá devido aos efeitos de eventos extremos em diversos setores da economia, da agricultura, da saúde publica e setores sociais. Desta forma apresentamos um modelo baseado em redes neurais artificiais para aprendizagem dos padrões do Histograma de Gradientes Orientados em imagens de satélite buscando identificar a ocorrência ou não de eventos extremos.

Palavras-chave. Eventos extremos, imagens de satélite, redes neurais artificiais, Histograma de Gradientes Orientados.

\section{Introdução}

Eventos climáticos, principalmente os considerados extremos, como precipitações de grandes proporções, ciclones, tornados e tsunamis, são determinantes no comportamento econômico e social, pois acarretam em grandes prejuízos. As mudanças climáticas são um fator determinante para o aumento da frequência de eventos climáticos extremos [7], identificar e emitir alertas em um curto período de tempo para tomada de decisões é um desafio.

\footnotetext{
${ }^{1}$ luisricardoengcomp@gmail.com

${ }^{2}$ eng.emersonjsilva@gmail.com

${ }^{3}$ francisca.nascimento@inpe.br

${ }^{4}$ lamar.guima@gmail.com

${ }^{5}$ rrrosa.inpe@gmail.com
} 
A análise destes eventos pode ser feita de maneira visual indicando nas imagens de satélites regiões propensas a ocorrência de eventos extremos. Entretanto, avaliar um grande volume de dados gerados diariamente é uma tarefa não-trivial, ainda mais, quando é necessária a avaliação de especialistas para indicar a ocorrência destes eventos. Neste sentido, apresentamos um modelo de redes neurais de múltiplas camadas para identificação dos padrões do Histogram of Oriented Gradients (HOG) em imagens de satélites de eventos extremos executando uma classificação binária para identificar a ocorrência destes eventos.

\section{Redes Neurais e o Modelo Multilayer Perceptron (MLP)}

De maneira geral redes neurais artificiais (RNAs) são modelos computacionais que se baseiam no sistema nervoso de um organismo (seres humanos e animais), tentando aproximar o processamento de informações de forma similar ao cérebro, este modelo é altamente difundido em problemas de reconhecimento de padrões [3]. Um dos modelos de RNAs é o modelo Multilayer Perceptron (MLP), que consiste em um conjunto de unidades de processamento (neurônios) conectadas e dispostas em uma topologia de múltiplas camadas para o processamento de padrões de entrada $\left\{x_{1}, x_{2}, \ldots, x_{n}\right\}$ [8]. Estas unidades são regidas por uma regra de aprendizado $(\delta)$ que permite a adaptação dos pesos $\left\{w_{1}, w_{2}, \ldots, w_{j}\right\}$ para executar uma tarefa de classificação, ou seja, mapear os valores de $\left\{x_{1}, x_{2}, \ldots, x_{j}\right\}$ para suas respectivas classes $\left\{y_{1}, y_{2}, \ldots, y_{j}\right\}$. Este modelo possui camadas intermediárias que se adaptam a problemas não lineares, realizando um processamento dos dados e ajustando os erros antes de propagar para a camada de saída este ajuste se dá pelo algoritmo de retropropagação de erro (backpropagation). O algoritmo de treinamento backpropagation é [4] dado pela regra delta. O cálculo do valor do erro e a regra delta são descritos respectivamente em (1) e (2).

$$
\begin{gathered}
e_{j}=d_{j}-y_{j} \\
\delta_{j}=e_{j} \dot{\varphi}_{j}\left(v_{j}\right)
\end{gathered}
$$

Onde $e_{j}$ é o erro do neurônio $\mathrm{j}, d_{j}$ é o resultado esperado da ativação do neurônio (o padrão o qual o neurônio representa) e $y_{j}$ é o resultado obtido. Em (2) $\delta_{j}$ é o delta do neurônio j e $\dot{\varphi}_{j}\left(v_{j}\right)$ é derivada da função de ativação do neurônio j. Em (3) é descrito o somatório para o cálculo do delta feito entre as camadas intermediárias de neurônios conectados $[4,6]$.

$$
\delta_{j}=\dot{\varphi}_{j}\left(v_{j}\right) \sum_{k} \delta_{k} w_{k j}
$$

Inicialmente o treinamento é feito pela propagação do erro para as camadas intermediárias (para frente), onde são calculados os valores de erro e do delta. Em seguida estes valores são derivados e propagados para as camadas anteriores (para trás) e $w_{i}$ são atualizados. Cada época de treinamento avalia o conjunto de dados por completo, são necessárias $n$ épocas até que a RNA atinja um limiar ou um critério de parada, geralmente isto se dá pelo menor índice possível de erro na classificação. 


\section{Histogram of Oriented Gradients - HOG}

Uma das maneiras de avaliar as principais características de imagens é o Histograma de Gradientes Orientados (HOG), esta técnica permite que se obtenham apenas informações importantes das imagens diminuindo a complexidade de análise [2].

Esta técnica foi desenvolvida por [1], para detecção de objetos em imagens principalmente voltadas ao reconhecimento facial. A ideia fundamental do HOG é identificar as características de borda e formatos nas imagens através da distribuição dos gradientes de intensidade dos pixeis e pelas direções das bordas. O gradiente é dado por (4), e é referente ao vetor das derivadas parciais dos pontos $x$ e $y$.

$$
\nabla f=\left[\begin{array}{l}
g_{x} \\
g_{y}
\end{array}\right]=\left[\begin{array}{l}
\frac{\partial f}{\partial x} \\
\frac{\partial f}{\partial y}
\end{array}\right]
$$

Após o calculo dos gradientes é aplicado um filtro na imagem a para abstração das características de cor e intensidade. Para o cálculo do HOG uma imagem é dividida em blocos, ou regiões, por exemplo, em matrizes de 16x16 pixeis. Para cada um destes blocos a magnitude do gradiente e um número de direções é calculado conforme (5) e (6) respectivamente [1].

$$
\begin{gathered}
g=\sqrt{g_{y}^{2}+g_{x}^{2}} \\
\theta=\arctan \left[\begin{array}{l}
g_{y} \\
g_{x}
\end{array}\right]
\end{gathered}
$$

O HOG transforma uma imagem RGB em um vetor de características (a distribuição e as direções dos gradientes na imagem). Os gradientes são úteis pois a magnitude dos gradientes é elevada em torno das bordas dos objetos, desta forma essas bordas possuem mais informações sobre os objetos presentes na imagem. A Fig. 1 demonstra o cálculo do HOG para uma imagem com ocorrência de um evento extremo.
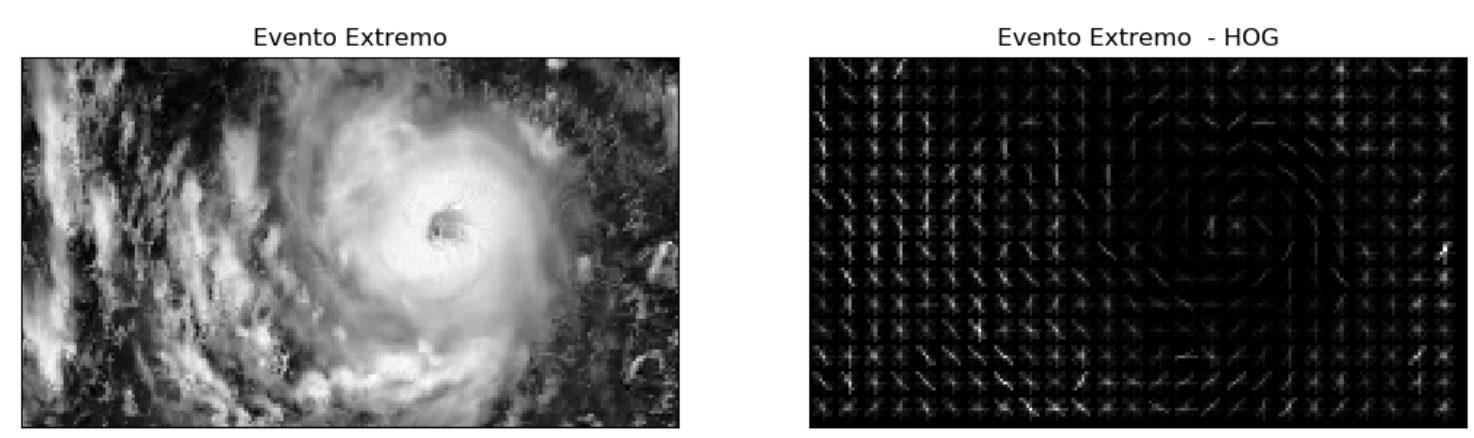

Figura 1: Histograma de gradientes orientados para eventos extremos

Desta forma, com os vetores HOG calculados para as imagens foi possível gerar um conjunto de treinamento $(x)$ e definir as saídas $(y)$ de não-evento e evento $(0,1)$. 


\section{Descrição dos Dados}

As imagens obtidas neste trabalho são divididas em imagens com eventos e sem eventos extremos. As imagens possuem uma resolução espacial variando entre $10 \mathrm{~km}$ a $20 \mathrm{~km}$ no canal infravermelho, Banda 4 infravermelho termal e foram selecionadas do acervo do INPE [5] do satélite LANDSAT-8, e do acervo do NOAA [9] do satélite GOES, os dados foram coletados em Julho de 2018. Para gerar um vetor de características que fosse capaz de identificar os eventos em regiões isoladas as imagens foram segmentadas em blocos com resoluções variadas para identificar os eventos, conforme ilustra a Fig. 2. As imagens sem a ocorrência de eventos possuem cobertura de nuvens mínima, evitando a análise de um falso positivo.
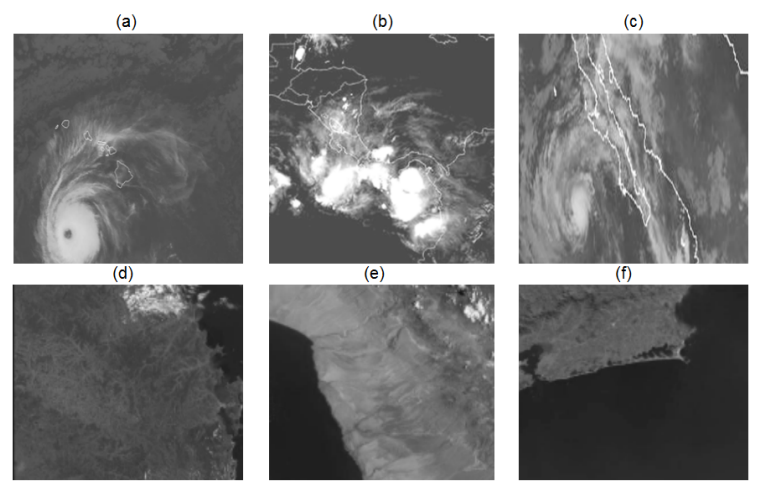

Figura 2: Imagens de satélite com eventos e sem eventos. Em (a,b,c) são dispostas as imagens com exemplos de eventos extremos enquanto (d,e,f) representam a ausência destes eventos.

Para obter um conjunto de dados amplo foram geradas imagens artificiais para o treinamento do modelo. As imagens artificiais variam em rotação, inclinação e posição de forma a gerar sobre uma imagem original diversas variações da mesma. A Fig. 3 ilustra algumas imagens artificiais geradas neste trabalho.
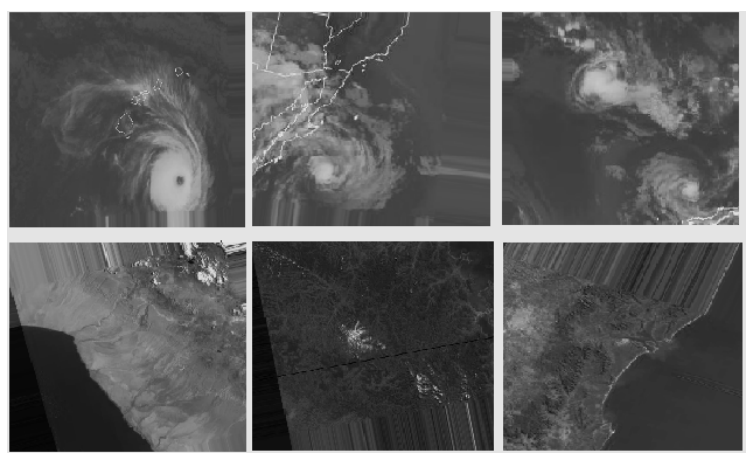

Figura 3: Imagens artificiais.

Foram geradas um total de 1810 imagens (50\% para evento e 50\% para não-evento) para o treinamento e teste RNA. 


\section{Treinamento do Modelo}

As 1810 imagens de eventos foram submetidas a um pré-processamento de dados que visou redimensionar todas as imagens em um único padrão de 300x300 pixeis, e transformar as imagens do canal RGB para escala de cinza. Após esta normalização foi calculado o HOG (em blocos de 12x12 pixeis) de cada imagem, gerando assim um vetor de características. O treinamento da rede MLP seguiu as configurações descritas na Tab. 1.

Tabela 1: Topologias de treinamento testadas no modelo MLP

\begin{tabular}{cccccc}
\hline Configuração & Camadas & Neurônios & Configuração & Camadas & Neurônios \\
\hline Config. 1 & 2 & 2 & Config. 7 & 3 & 12 \\
Config. 2 & 2 & 4 & Config. 8 & 3 & 25 \\
Config. 3 & 2 & 12 & Config. 9 & 4 & 2 \\
Config. 4 & 2 & 25 & Config. 10 & 4 & 4 \\
Config. 5 & 3 & 2 & Config. 11 & 4 & 12 \\
Config. 6 & 3 & 4 & Config. 12 & 4 & 25 \\
\hline
\end{tabular}

O modelo MLP utilizado neste trabalho foi treinado utilizando a função de ativação 'ReLU' (Ativação linear retificada) e sua derivada dada por (7) e obedeceu um critério para escolha da melhor topologia para o treinamento da RNA.

$$
\operatorname{ReLU}(x)=\max \{0, x\} \quad \operatorname{Re} L U^{\prime}(x)= \begin{cases}1, & \text { se } x \geq 0 \\ 0, & \text { c.c. }\end{cases}
$$

O critério foi o incremento de camadas e a variação gradativa de neurônios em cada camada observando os valores de erro até atingir um valor mínimo. Este critério foi escolhido devido a capacidade da RNA em separar problemas não lineares, ou seja, quanto mais camadas e neurônios mais "retas" no hiperplano de parâmetros existem para discriminar o problema. A Fig. 4 indica o comportamento do treinamento para as configurações da Tab. 1 indicando qual configuração atinge o menor erro em um determinado número de épocas.
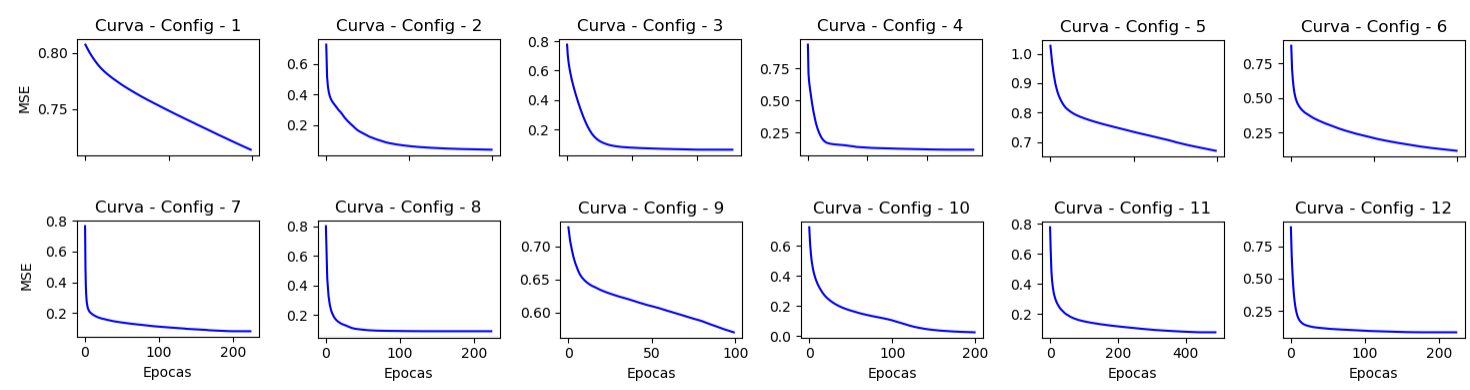

Figura 4: Decaimento do erro quadrático durante o treinamento da Rede MLP. 
Foram utilizadas $70 \%$ das imagens para o treinamento e 30\% das imagens para a validação do modelo. A melhor configuração foi a Config. 8 com 3 camadas intermediárias com 25 neurônios cada. Esta configuração atingiu o erro médio quadrático de 0.104.

\section{Resultados e Conclusões}

Os resultados obtidos com a abordagem são descritos na Tab. 2. Os testes foram feitos para 544 imagens sendo $50 \%$ para eventos e $50 \%$ para não-eventos.

Tabela 2: Resultados de Classificação de Eventos Extremos

\begin{tabular}{cccc}
\hline Acurácia & Precisão & Recall & F1-Score \\
\hline 0,895 & 0,897 & 0,895 & 0,894 \\
\hline
\end{tabular}

A Fig. 5 indica o comportamento do modelo no treinamento e na validação cruzada utilizando diferentes amostragens para o conjunto de treinamento. A curva revela que o modelo generaliza o conjunto de treinamento em quase $99 \%$ das amostras, para o conjunto de teste obtém-se 89,5\% na eficiência da classificação. Os índices de precisão indicam o desempenho na classificação binária entre evento extremo e não-evento em $89 \%$ das amostras.

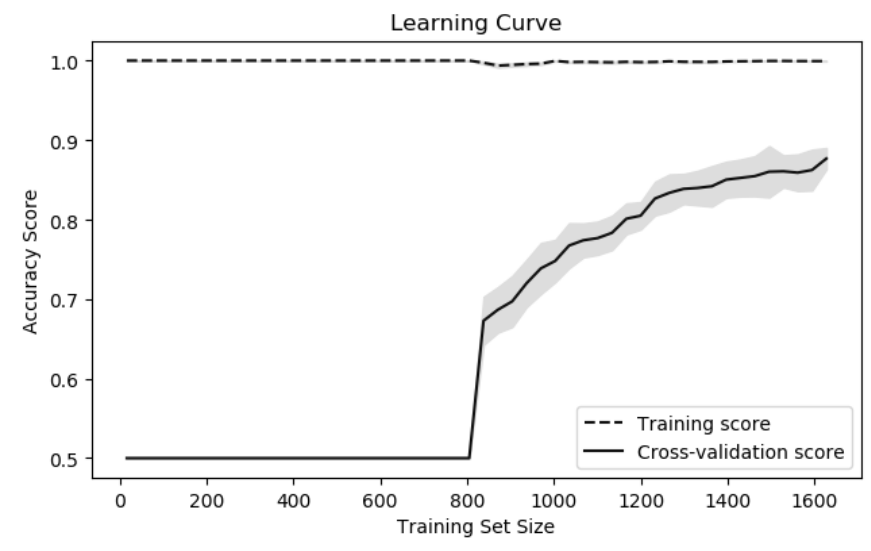

Figura 5: Curvas de Aprendizado do Modelo MLP. A região cinza indica o desvio padrão dos scores obtidos no decorrer do treinamento.

É importante ressaltar que a proposta apresentada neste trabalho não é definitiva, a generalização em $89 \%$ para correta classificação dos eventos extremos é uma aproximação inicial. A estrutura gerada neste trabalho permite alternativas a otimização e variação dos modelos de machine learning para o treinamento e testes de classificação. A curva apresentada na Fig. 5 indica que houve um crescimento na acurácia da validação do modelo conforme o tamanho do conjunto de treinamento aumenta, isto significa que quanto mais a rede avalia os padrões de eventos extremos maior é a precisão do modelo. 


\section{Agradecimentos}

Agradecemos ao CNPq e à CAPES pelo suporte financeiro. Agradecemos ao INPE e ao IEAv pelo apoio à pesquisa e suporte computacional.

\section{Referências}

[1] N. Dalal, B. Triggs. Histograms of oriented gradients for human detection. In: international Conference on computer vision \& Pattern Recognition $\left(C V P R^{\prime} 05\right)$. IEEE Computer Society, 2005. p. 886-893.

[2] G. L. David. Distinctive image features from scale-invariant keypoints. International journal of computer vision, v. 60, n. 2, p. 91-110, 2004.

[3] L. Fausett. Fundamentals of neural networks: architectures, algorithms, and applications. Prentice-Hall, Inc., 1994.

[4] S. Haykin. Redes neurais: princípios e práticas. Bookman, Porto Alegre, 2001.

[5] INPE - Instituto Nacional de Pesquisas Espaciais. Catálogo de Imagens. 2018. Disponível em: http://www.dgi.inpe.br/CDSR/ Acesso em: 15 de julho de 2018.

[6] G. F. Luger. Inteligência Artificial. Pearson Education do Brasil, São Paulo, 2013.

[7] S. Mal, R. B. Singh, C. Huggel (Ed.). Climate Change, Extreme Events and Disaster Risk Reduction: Towards Sustainable Development Goals. Springer, 2017.

[8] W. S. Mcculloch, W. Pitts.A logical calculus of the ideas immanent in nervous activity. The bulletin of mathematical biophysics, v. 5, n. 4, p. 115-133, 1943.

[9] NOAA - National Oceanic and Atmospheric Administration. Geostationary Satellite Server. 2018. Disponível em: http://www.goes.noaa.gov/browsw.html Acesso em: 15 de julho de 2018 . 